\title{
Integrated cardiothoracic surgery: Navigating interviews and the match
}

\author{
Benjamin Smood, MD, ${ }^{a}$ Stephanie N. Nguyen, MD, ${ }^{b}$ Abigail R. Benkert, MD, ${ }^{c}$ and Jason J. Han, MD $^{\mathrm{a}}$
}

We previously provided recommendations to assist aspiring cardiothoracic surgeons in the process of developing competitive applications for integrated cardiothoracic surgery residency programs (IPs). ${ }^{1}$ We hope this Young Surgeon's Note can serve as a foundation for individuals when applying to IPs and developing a rank order list (ROL). Several online resources exist for applicants interested in IPs, ${ }^{2-4}$ including program websites, but these frequently lack additional information that may be pertinent to applicants' decision making. ${ }^{5}$ Here we explore strategies to maximize applicants' interview opportunities and their ability to succeed in the National Residency Matching Program, while highlighting important considerations for candidates as they determine where to apply and how to develop their ROL. These recommendations reflect our personal experiences in recent application cycles and incorporate solicited feedback from other resident physicians. Of course, no algorithm exists to guarantee that an applicant will match with his or her first-choice program, and readers certainly should not interpret these suggestions as the modus operandi of all applicants who match into IPs. Rather, we hope this Young Surgeon's Note can serve as a peer guide for aspiring cardiothoracic surgeons, particularly for those who may not have access to other mentors familiar with the IP application process.

\section{SETTING ONESELF UP FOR A SUCCESSFUL MATCH}

\section{Dual Applying to IP and General Surgery Programs}

Even outstanding applicants may face challenges matching into IPs given the limited number of positions and the unpredictability of the match. ${ }^{1}$ One survey of IPs found that $60 \%$ of programs received applications from nearly all applicants in the IP pool, but interviewed only 20 or 30. ${ }^{6}$ In 2018, 170 IP applicants (99 US medical graduates and 71 international medical graduates) applied for 36 IP positions. Programs received an average of 114 out of 170 (67\%) applications $(69 \%$ US medical graduates and $65 \%$ international medical graduates). ${ }^{7}$ In total, 31 out of $36 \mathrm{IP}$ positions $(86 \%)$ were filled by US medical graduates,

\footnotetext{
From the ${ }^{\text {a Division }}$ of Cardiovascular Surgery, Department of Surgery, The University of Pennsylvania, Philadelphia, Pa; ${ }^{\mathrm{b}}$ Division of Cardiac, Thoracic, and Vascular Surgery, Department of Surgery, New York Presbyterian/Columbia University Medical Center, New York, NY; and ${ }^{\mathrm{c} D i v i s i o n}$ of Cardiovascular and Thoracic Surgery, Department of Surgery, Duke University Medical Center, Durham, NC.

Disclosure: Authors have nothing to disclose with regard to commercial support.

Received for publication Jan 11, 2020; revisions received Feb 3, 2020; accepted for publication Feb 4, 2020; available ahead of print Feb 25, 2020.
}

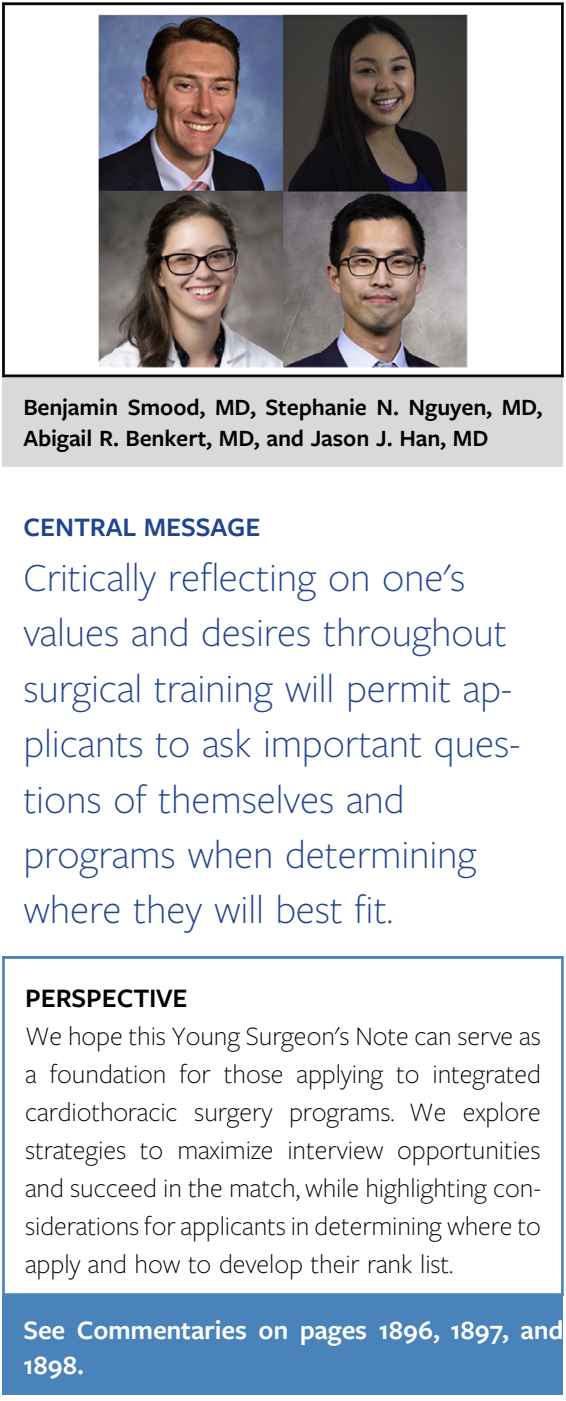

painting a highly competitive landscape even for this group filling the majority of positions. ${ }^{8}$ Nevertheless, applicants should be reassured that they can still receive excellent training and become cardiothoracic surgeons via a general surgery (GS) residency with either a $4+3$ joint surgery/

Address for reprints: Benjamin Smood, MD, Division of Cardiovascular Surgery, Department of Surgery, University of Pennsylvania, 3400 Spruce St-4 Maloney, Philadelphia, PA 19104 (E-mail: benjamin.smood@pennmedicine.upenn.edu). J Thorac Cardiovasc Surg 2021;161:1889-95 0022-5223/ $\$ 36.00$

Copyright (C) 2020 by The American Association for Thoracic Surgery https://doi.org/10.1016/j.jtcvs.2020.02.074 
thoracic surgery program or a traditional cardiothoracic fellowship. ${ }^{5}$ As such, dual applying to both IPs and categorical GS programs is a prudent strategy utilized by more than $90 \%$ of IP applicants. ${ }^{9,10}$ Very few applicants apply solely to IPs, and this is increasingly uncommon (only 1 US applicant and 6 international medical graduates in 2018). ${ }^{8}$ Although some successfully match into IPs, we strongly discourage this strategy.

Developing a unique GS application can be difficult for IP candidates whose early commitments to cardiothoracic surgery likely embody a large portion of the experiences and accolades within their applications. Some applicants believe that applying to IPs can diminish their chances of matching into GS programs. ${ }^{6,11}$ Certainly, relying too heavily on cardiothoracic surgery merits can convey a single-track mindset to GS program directors, and may be viewed as a neutral or even negative trait. ${ }^{11}$ However, this need not be the case. We believe that expressing interest in cardiothoracic surgery while applying to GS programs can be done judiciously by preparing 2 appropriately directed personal statements and soliciting separate recommendation letters for the specialties. Demonstrating a record and commitment to excellence in academic surgery, regardless of specialty, highlights one's acumen and work ethic and is generally attractive to programs.

\section{Determining the Number of Programs That Should Receive Applications}

There is a strong impetus for candidates to apply to a high number of programs, including GS, to avoid the risk of not matching at all. However, there is a point at which applying to additional programs becomes superfluous. For US medical graduates with competitive Board scores $(\geq 239)$, the benefit of applying to additional programs diminishes at about 36 GS applications. ${ }^{12}$ Unfortunately, similar data regarding diminishing returns is not available for IP applicants. Most dual-program applicants who successfully match into IPs apply to more than 30 non-IPs (ie, usually GS) in addition to about 20 IPs (Figure 1). ${ }^{7,10}$ We believe this combination is generally reasonable and sufficient, but some argue that applicants should apply to every IP because interviews can always be declined once offered. Certainly, applying to residency requires honest reflection of one's competitiveness to succeed in the match. ${ }^{1}$ Highly competitive IP candidates may be comfortable applying to fewer GS programs, just as less-competitive applicants may feel the need to buffer their application with more IP and GS applications. ${ }^{6}$

Most applicants will not secure an interview at every program to which they apply. However, statistics have been generally encouraging. Among all GS applicants, 90\% match into the specialty when ranking 11 programs. ${ }^{13}$ Although similar data are not published for IP applicants, with these caveats and perspectives, we suggest that applicants aim to interview at and rank about 20 programs in total. It is not uncommon to attend 10 to 15 IP interviews in addition to $10 \mathrm{GS}$ interviews, ${ }^{6}$ but attending so many GS interviews may be excessive for candidates who receive a significant number of IP interview invitations because having a higher proportion of IP interviews portends a higher likelihood of matching into an IP. Thus, although it is important to apply broadly, realize that once interview invitations are sent, there are significant costs and logistical challenges associated with interviewing. Moreover, applicants can underestimate the demands of repetitive interviews. Exhaustion may be reflected in interview performance and
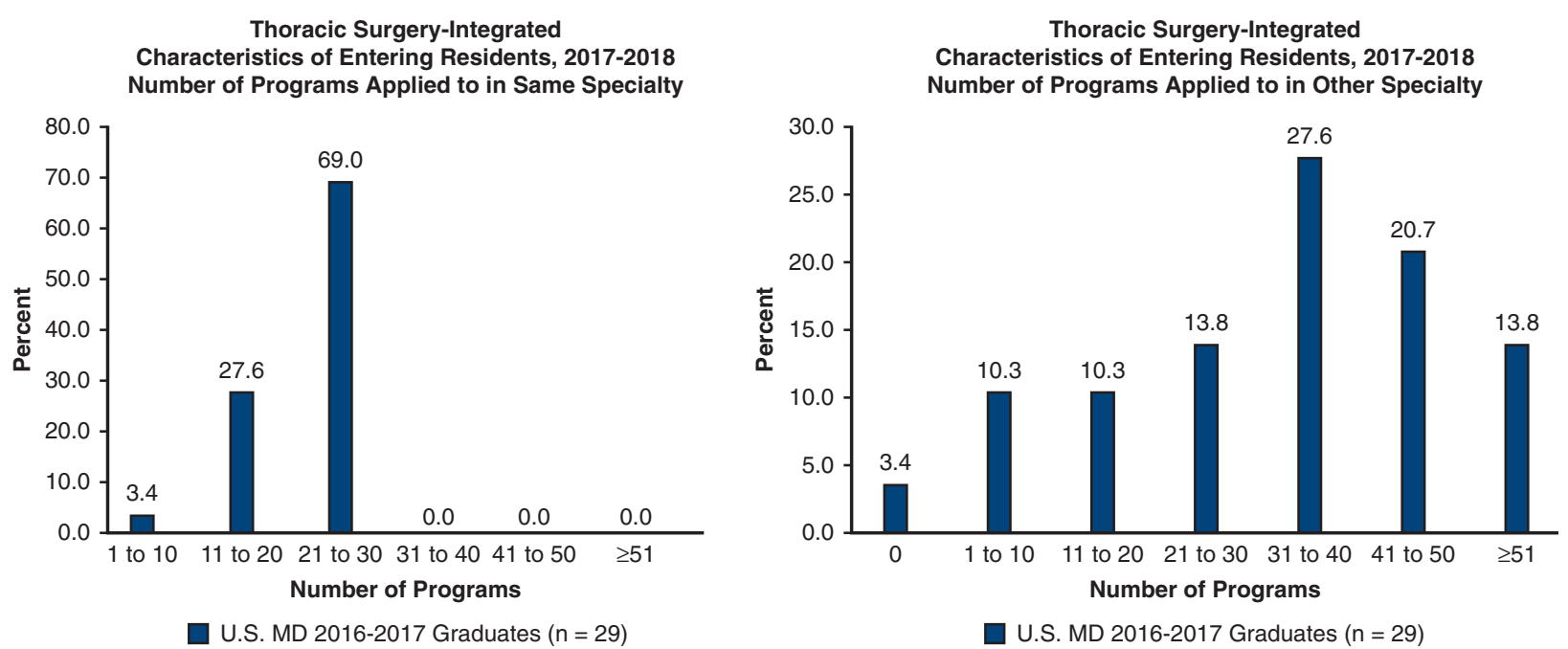

FIGURE 1. Left, Number of integrated cardiothoracic surgery programs that United States medical graduates applied to, among residents entering integrated cardiothoracic surgery residency. Incoming integrated cardiothoracic surgery residents, on average, applied to 22 integrated cardiothoracic surgery programs. Right, Number of programs applied to in other specialties by applicants entering integrated cardiothoracic surgery residency. On average, United States medical graduates applied to 37 additional programs. Reproduced with permission. ${ }^{10}$ 
unintentionally perceived as a lack of enthusiasm. To minimize interview fatigue, applicants should only interview at programs where they genuinely and eagerly envision themselves training.

\section{INTERVIEWS}

\section{Receiving Invitations and Scheduling Interviews}

One can expect to begin receiving IP interview offers as early as October; however, some invitations may be delayed through November or even December for interviews set to take place in January or February. Some applicants will receive invitations from more programs than they are logistically able to attend. Because IP interview dates frequently overlap across institutions, one should develop a strategy to avoid scheduling conflicts. This can be difficult because some programs offer only 1 interview date, whereas others offer multiple dates on a first-come-first-served basis. Therefore, make every effort to respond to interview invitations as soon as possible to reserve your desired date. Two tools can be helpful in developing a strategy to maximize the number of interviews you can attend.

First, the Thoracic Surgery Directors Association publishes many of the previous year's interview dates online (and in some instances, dates for the upcoming interview season). ${ }^{4}$ About one-third of IPs publish interview dates on their division websites. ${ }^{5}$ It is prudent, albeit time consuming, for applicants to develop a master calendar of the past year's interview dates (and future dates if listed) because individual programs frequently interview around the same time each season. With these data, applicants can anticipate potential conflicts, which can be particularly helpful to determine which days to select when multiple interview dates are offered at a program.

A second tool that can be useful is creating a unique email address for the match and all application-related correspondences. Many applicants will be in the midst of demanding visiting surgical electives when they receive invitations. Providing a trusted confidant with access to this email account will enable them to secure interviews if an applicant is scrubbed into cases on subinternships when invitations are sent. Sharing the aforementioned master calendar and a detailed list of prioritized programs and interview dates can help ensure that pending interviews are left available should applicants be invited, particularly when only a single date is expected to be offered. Although one may be able to schedule an interview while on a demanding rotation, note that traveling for interviews becomes exceptionally busy in early- to mid-November and through January. We recommend completing electives before November so that applicants can focus on interviews.

\section{Interviewing}

Ideally, interviewing should not be an anxietyprovoking experience. Applicants should reflect on and take pride in having been chosen from a talented and self-selecting pool of IP applicants. Interviews are the culmination of years of hard work and the opportunity of a lifetime to demonstrate why a program would enjoy spending the better part of a decade training an applicant. To a large extent, what follows is a determination of fit. ${ }^{14}$ This intangible synergism acts to facilitate collaboration among like-minded institutions and residents, which provides an ideal environment to maximize surgical training and education. Of course, applicants should also be prepared to talk about anything on their application in detail, including why they have chosen this career path and their future aspirations. To reiterate, in considering IPs to which to apply, individuals should think about why they are applying to each because this is an important point to articulate during interviews. Programs are looking for attributes that will help a trainee thrive in the field, and more specifically, in the culture and environment of their institution. It is important for applicants to remember that this is also their opportunity to gather as much information as possible about a program, which will ultimately factor into developing their ROL.

\section{CREATING AN ROL}

Each applicant values different attributes to varying degrees when developing a ROL, and it is difficult to find programs that are a perfect fit in all facets. Conversing with current trainees is an integral part of interviews that offers unique vantage points for understanding the nuances in culture and curriculum among residency programs. Candid discussions regarding education and life outside of the hospital can help applicants assess elements that hold significant value in determining a program of best fit. Developing a system to recall these aspects months later can help finalize one's ROL. Here we explore several program attributes that are heterogeneously valued by trainees so that applicants can be prepared to ask pertinent questions of programs and themselves (Table 1). These are discussed in no order of importance, as each factor will be weighted with respect to each applicant's preferences.

\section{Variety in Cardiac and Thoracic Training}

Applicants that anticipate a future as joint cardiac and thoracic surgeons or remain open-minded in their career aspirations must ensure that their operating experience will be particularly well rounded. Some IPs consciously dedicate equivalent time to cardiac and thoracic rotations throughout the entirety of senior fellowship years. Applicants committed to specializing in either cardiac or thoracic surgery might prefer institutions that offer a broad initial exposure to both areas, but ultimately facilitate an elected focus as they advance in their training. Most programs publish rotation schedules on their websites. ${ }^{5}$ Although 
TABLE 1. Considerations for integrated cardiothoracic surgery applicants when applying to residency programs and developing a rank order list

\begin{tabular}{|c|c|}
\hline Variable to consider & Supporting points \\
\hline Variety in cardiac and thoracic training & $\begin{array}{l}\text { - Equivalent time dedicated to thoracic and cardiac rotations } \\
\text { - Emphasized focus on thoracic surgery or cardiac surgery } \\
\text { - Ability to elect a focus in cardiac or thoracic surgery } \\
\text { - Surgical exposure variety } \\
\text { - Territory of foregut operations (thoracic vs general surgeons) } \\
\text { - Minimally invasive/video-assisted vs open procedures } \\
\text { - Robotic surgery } \\
\text { - Transcatheter techniques } \\
\text { - Subspecialty exposure (congenital, transplantation, other) }\end{array}$ \\
\hline Operative experience: Volume and autonomy & $\begin{array}{l}\text { - Total operative volume of program and capacity to perform cases } \\
\text { - Complexity and variety of cases } \\
\text { - Resident operative volume and capacity to cover cases } \\
\text { - Resident autonomy } \\
\text { - Components of cases performed at varying training levels } \\
\text { - Difficulty meeting any graduation case requirements } \\
\text { - Adherence to } 80 \text {-h work week } \\
\text { - Presence of } 4+3 \text { residents or traditional fellows }\end{array}$ \\
\hline $\begin{array}{l}\text { Nonoperative resident duties: Clinic } \\
\text { experience and patient management }\end{array}$ & $\begin{array}{l}\text { - Clinic requirements } \\
\text { - Role in fielding consults and preoperative evaluation } \\
\text { - Postoperative management in intensive care units and on wards } \\
\text { - Advanced practice providers and supporting staff } \\
\text { - Non-operative responsibilities during senior fellowship years }\end{array}$ \\
\hline Training sites & $\begin{array}{l}\text { - Presence of Veterans Administration hospital } \\
\text { - Exposure to community/private practice models } \\
\text { - Number of hospitals required to cover } \\
\text { - Distance between rotation sites }\end{array}$ \\
\hline Academic pursuits & $\begin{array}{l}\text { - Emphasis toward training academic surgeon-scientists } \\
\text { - Supportive of careers in private practice } \\
\text { - Requirements or inability to take dedicated research time } \\
\text { - Flexibility in time allotment for academic pursuits } \\
\text { - Flexibility in character of academic pursuits (clinical fellowships, travel to other institutions) } \\
\text { - Advanced degrees offered } \\
\text { - Funding provided for research } \\
\text { - Support for presenting research at conferences }\end{array}$ \\
\hline Fellowships & $\begin{array}{l}\text { - Offer fellowships specific to personal career aspirations } \\
\text { - Accessible mentors in desired field of specialty }\end{array}$ \\
\hline Work-life balance and community & $\begin{array}{l}\text { - Geographic preferences } \\
\text { - Outdoor activities } \\
\text { - Housing prices } \\
\text { - Job opportunities for spouses } \\
\text { - Local schools } \\
\text { - Familial status of colleagues } \\
\text { - Sense of community amongst integrated program residents }\end{array}$ \\
\hline Institutional culture & $\begin{array}{l}\text { - Frequency and structure of didactics } \\
\text { - Formal mentorship } \\
\text { - Resident-faculty dynamics } \\
\text { - Culture of feedback and operating room environment } \\
\text { - Faculty fluidity }\end{array}$ \\
\hline
\end{tabular}

proportionally more IP applicants envision a career in cardiac surgery, ${ }^{15}$ those interested in thoracic surgery can find suitable programs.
Applicants interested in thoracic surgery should evaluate the variety of procedures, including the prevalence of open thoracotomies, as well as robotic and/or video-assisted 
thoracoscopic surgery. Determine whether thoracic surgeons perform the majority of foregut operations at the institution, because this area is often shared with general surgeons. ${ }^{14}$ In this respect, applicants interested in thoracic surgery may prefer to rank some GS programs higher than IPs to develop an excellent foundation in abdominal and foregut surgery. ${ }^{16}$ However, when determining how to rank GS programs, consider whether programs offer cardiothoracic rotations during residency.

Whereas IPs necessarily provide adequate exposure to both cardiac and thoracic surgery for fulfilling graduation requirements, some programs have a concentrated emphasis on cardiac surgery. ${ }^{14,17}$ Applicants interested in a specific area of subspecialization (eg, heart failure/mechanical circulatory support, aortic and endovascular/transcatheter, or congenital) should identify programs with strong exposure in their desired field. ${ }^{17}$ Graduating IP trainees may not need to pursue additional fellowships after residency, and can even direct transplant or transcatheter programs if they have accrued sufficient experience throughout their training. Determining whether graduates meet such benchmarks is a prudent consideration for individuals considering subspecialization. Ultimately, surgical education should fit one's desired career goals while being sufficiently broad to ensure trainees remain marketable upon graduation in any field because interests may change.

\section{Operative Experience: Volume and Autonomy}

There is considerable heterogeneity in resident operative experiences among IPs. ${ }^{18}$ Evaluate programs in part based on factors such as operative volume, variety of cases, and the degree of resident autonomy. Operative experience and volume can be best assessed by speaking with residents in their senior fellowship years of IPs during that trainees accumulate the lion's share of their cardiothoracic surgical training. Simple metrics for operative volume include how many cases senior residents perform per week, viewing residents' total case logs at the time of graduation, and if there are any difficulties in meeting requirements. One can also assess how programs introduce graduated responsibilities over time by establishing when trainees are introduced to various operating components such as cannulation, harvesting internal thoracic artery, and sewing distal anastomoses. In evaluating autonomy, assess aspects of the institution's educational culture, such as how often fellows are on the surgeon's side of the table. Keep in mind that assisting and performing cases as the primary surgeon, at varying levels of complexity, can offer uniquely different challenges and lessons to trainees. ${ }^{19}$ Of course, autonomy will vary with respect to case complexity, a resident's technical level, and the attendings' willingness to assist. Nevertheless, patterns can be gleaned from asking the right questions.

An important factor to consider is the ratio between operative volume and resident capacity. If an institution's operative volume far exceeds resident capacity to cover cases, this may demand frequent coverage into the evenings, overnight, and on weekends. Although some IPs require in-house call, others allow call from home. Residents are often required to be available for transplant procurements, whereas others make this optional. Although some trainees may thrive on the notion of routinely surpassing the 80-hour work week, recognize that this practice is uncommon, ${ }^{15}$ and training programs are committed to protecting residents' well-being, particularly with respect to work-hour regulations. Moreover, a resident can only be 1 place at a time, and thus there are practical limitations for how many cases in which an individual can feasibly participate, no matter how much volume an institution has. Also consider that larger IPs with traditional cardiothoracic fellows may provide relieving coverage, but can also reduce the number of cases in which IP residents take part. Furthermore, there can be institutional limitations on how many cases can logistically be performed each day if supporting staff, intensive care units, or operating room availability are limited. Therefore, it can be valuable to understand how programs anticipate evolving in the coming years. Recognize that additional factors beyond just potential operating experience contribute to one's education and development as a surgeon.

\section{Nonoperative Resident Duties: Clinic Experience and Patient Management}

Although operative experience is of paramount importance, other facets of surgical education must also be considered, including clinic requirements and perioperative patient management. The degree to which trainees are involved in these duties varies across institutions, ${ }^{18}$ but can be difficult to assess before interviews. ${ }^{5}$

On one end of the spectrum, residents may be largely responsible for decisions and care in the intensive care unit, on the floor, and/or be required to attend clinic multiple times per week. Developing such autonomy is essential for the clinical acumen necessary to managing patients as an attending surgeon. However, nonoperative duties, particularly during senior fellowship years, can also interrupt chances to spend time in the operating room.

At the other end of the spectrum, some IPs have minimal monthly clinic requirements and an infrastructure in place such that moment-to-moment perioperative care is largely directed by a team of intensivists and advanced practice providers. When clinics and surgical units function independently of residents, trainees may spend more time in the operating room. However, in these instances, discipline and ownership of one's education are required to become competent in the nonoperative aspects of surgical care. Understanding the structure and direction provided at varying IPs, and how applicants value these models in light of their own preferred learning 
environments, is an important consideration when evaluating programs.

\section{Training Sites}

A unique opportunity for balancing clinical and operative duties can be found at institutions with Veterans Administration (VA) hospitals, which are often resident-run services in which trainees are involved in every aspect of care from initial presentation in clinic and as consults, determining whether surgery is indicated, scheduling and performing operations, and managing patients postoperatively. Many residents believe these are among the best rotations for developing decision-making skills. However, the VA tends to have lower operative volume than large academic centers, so whereas there is considerable administrative autonomy, operative experiences may be comparatively diminished. Applicants should also determine whether residents are required to cover multiple hospitals. Some find off-campus rotations particularly isolating early in their training, and others find that needing to provide their own transportation and commuting between various sites is burdensome. Conversely, private, community, and academic hospitals are vastly different, and offer residents unique operating experiences and vantage points regarding patient acuity, case complexity, and operative volume. Trainees may value this variety when considering a career in private versus academic practice.

\section{Academic Pursuits}

Some IPs prioritize training future academic surgeonscientists whereas others are equally supportive of careers in private practice. Determining whether dedicated research time is required, optional, or strictly forbidden is crucial in developing one's ROL. ${ }^{18}$ One should consider their potential academic ambitions, such as the number of years one would like to participate in research and their interest in earning an advanced degree during this time. It is important to know if programs are flexible because some applicants may decide that they want more or less academic time than what is expected. Applicants interested in international fellowships or more clinically focused fellowships (such as critical care) should inquire about a program's support for such endeavors during their academic years. In any case, determine whether programs provide funding for academic pursuits or whether residents are required to secure independent grants. Similarly, determine whether programs provide resources and leave time for presenting work at national or international conferences.

\section{Fellowships}

Although not essential, dedicated research time can be important for applicants interested in specific fellowships after graduation or subspecialized academic careers. For those considering additional training in light of their career aspirations, identify institutions that not only match residents into specialties of interest, but also those that offer such fellowships (eg, congenital, aortic, or transplantation). It is valuable to identify institutions that will foster relationships with mentors who can assist in a trainee's development and advocate on your behalf when it comes time to apply for fellowships.

\section{Work-Life Balance and Community}

Trainees should understand that well-being is an important part of a program's culture that may have a tremendous influence on your overall success. Of course, work-life balance must be taken from the perspective of an intense surgical training. Nevertheless, many applicants value the environment surrounding where they will be working. Geographic preferences may matter in ranking programs for any number of reasons, including nearby outdoor activities, the social scene, housing prices, job opportunities for spouses, and local schools if relevant. Although many programs have a broad mix of trainees with spouses and children, others attract candidates with fewer family commitments. In any case, trainees will spend more time with their coworkers than almost anyone else throughout their training, so consider whether a program makes a concerted effort to cultivate a distinct sense of community amongst integrated residents. The camaraderie experienced even during a brief interview day can provide important insight into relationships that can be anticipated.

\section{Institutional Culture}

Realize that educational communities also have unique personalities. Didactic conferences may be held multiple times per week and include structured curriculum and mentoring programs, whereas others consist of rare educational conferences and relatively informal mentorship. Some residents believe an emphasized culture of mentorship is essential to succeeding in IPs to complement the significant self-directed learning required of IPs. Dynamics between residents and faculty will vary. Although some institutions are clearly hierarchical, at others the residents are on a first-name basis with many attending surgeons.

Similarly, the culture in and around operating rooms may be extremely intense. Others have a calmer demeanor that still maintains a sense of urgency required for cardiothoracic surgery. In striving for excellence in performance and outcomes, resident feedback in the operating room can be harshly critical or delivered with more encouragement. Of course, this too will vary even among attending surgeons within a single institution, but applicants can ascertain a general sense of culture through interviews and should be assessed in light of one's own values and preferred learning environment. Applicants might consider revisiting for a second look after an interview if particularly 
drawn to a program, although these are not always permitted.

Applicants will inevitably interact with faculty they admire and those they believe will be ideal mentors for their training. These unique interpersonal relationships are essential to successful surgical training. However, applicants should not choose a program based solely on their relationship, experience, and/or expectation of a single individual. Leadership changes and faculty is fluid. It is important for applicants to be inspired and excited to train within the entirety of a program. Wherever an applicant matches, it can be expected that they will be satisfied ${ }^{15}$ and appreciate the program, their peers, and mentors with whom they dedicate so much of their lives.

\section{CONCLUSIONS}

We hope this Young Surgeon's Note helps guide applicants in evaluating the unique qualities of each program as they determine where they most want to train. Critically reflecting on one's values and desires throughout surgical training will permit applicants to ask the important questions of themselves and programs to determine where they would best fit as they develop their ROL. More than anything, individual efforts, discipline, and determination will enable trainees to finish residency well prepared and with the independence required to begin a career as a cardiothoracic surgeon.

\section{References}

1. Smood B, Nguyen SN, Kelly JJ, Han JJ. Young surgeon's note. Integrated cardiothoracic surgery developing a successful application. J Thorac Cardiovasc Surg. December 10, 2019 [Epub ahead of print].

2. American Medical Association. FREIDA residency and fellowship database. Available at: https://www.ama-assn.org/life-career/search-freida-online-amaresidency-fellowship-database. Accessed December 30, 2019.

3. Accreditation Council for Graduate Medical Education. Program search. Available at: https://apps.acgme.org/ads/Public/Programs/Search. Accessed December 30, 2019.
4. Thoracic Surgery Directors Association. Integrated thoracic surgery residency programs. Available at: http://www.tsda.org/the-tsda/ct-residency-programs/inte grated-residency-progams. Accessed December 30, 2019.

5. Miller VM, Padilla LA, Schuh A, Mauchley D, Cleveland D, Aburjania Z, et al Evaluation of cardiothoracic surgery residency and fellowship program websites. J Surg Res. 2020;246:200-6.

6. Karim AS, Meyerson SL. The residency application process: challenges for our specialty. J Thorac Cardiovasc Surg. 2017;153:1618-20.

7. Association of American Medical Colleges. ERAS statistics preliminary data Available at: https://www.aamc.org/data-reports/interactive-data/eras-statistics. Accessed December 30, 2019.

8. National Residency Matching Program. Results and data 2018 main residency match. Available at: http://www.nrmp.org/wp-content/uploads/2018/04/MainMatch-Result-and-Data-2018.pdf. Accessed December 30, 2019.

9. Association of American Medical Colleges. ERAS statistics cross specialty applicant data. Available at: https://www.aamc.org/system/files/reports/1/res dency-surgery.pdf. Accessed December 30, 2019.

10. Association of American Medical Colleges. Careers in medicine: thoracic surgery-integrated. Available at: https://www.aamc.org/cim/specialty/exploreop tions/list/us/341828/thoracic_surgery-integrated.html. Accessed December 30, 2019.

11. Meza JM, Rectenwald JE, Reddy RM. The bias against integrated thoracic surgery residency applicants during general surgery interviews. Ann Thorac Surg. 2015;99:1206-12.

12. Association of American Medical Colleges. Apply smart. Available at: https:// students-residents.aamc.org/applying-residency/filteredresult/apply-smart-dataconsider-when-applying-residency/. Accessed December 30, 2019.

13. National Residency Matching Program. Charting outcomes in the match: US allopathic seniors. Available at: https://www.nrmp.org/wp-content/uploads/2018/ 06/Charting-Outcomes-in-the-Match-2018-Seniors.pdf. Accessed December $30,2019$.

14. Woo YJ, Baker C, Colson Y, Cooke DT, Fann J, Goldstone A. Lessons learned: a roundtable discussion on succeeding in cardiothoracic surgical residency and practice. Semin Thorac Cardiovasc Surg. 2018;30:293-303.

15. Tchantchaleishvili V, LaPar DJ, Stephens EH, Berfield KS, Odell DD, DeNino WF. Current integrated cardiothoracic surgery residents: a Thoracic Surgery Residents Association survey. Ann Thorac Surg. 2015; 99:1040-7.

16. Tchantchaleishvili V, Barrus B, Knight PA, Jones CE, Watson TJ, Hicks GL. Sixyear integrated cardiothoracic surgery residency applicants: characteristics, expectations, and concerns. J Thorac Cardiovasc Surg. 2013;146:753-8.

17. Zhu Y, Goldstone AB, Woo JY. Integrated thoracic surgery residency: current status and future evolution. Semin Thorac Cardiovasc Surg. 2019:31:345-9.

18. Stephens EH, Walters DM, Eilers AL, Tchantchaleisvili V, Goldstone AB, Gillaspie EA, et al. Variability in integrated cardiothoracic training program curriculum. Ann Thorac Surg. 2017;103:1984-91.

19. Tolis G. Cardiac surgical training: a disincentivized necessity. Eur J Cardiothorac Surg. 2019;55:173-8. 